\title{
The Aging of Human-Immunodeficiency-Virus-Associated Neurocognitive Disorders
}

\author{
Tsuneya Ikezu
}

Received: 2 April 2009 /Accepted: 2 April 2009 /Published online: 5 May 2009

(C) Springer Science + Business Media, LLC 2009

This special issue proposes a new era for studies of the humanimmunodeficiency-virus-type-one (HIV-1) -associated neurological disorders serving to highlight cognitive abnormalities in infected aging patients. It has been 15 years since the "Third Workshop on NeuroAIDS" was held (Black and Sager 1994). At that time, NeuroAIDS (or the AIDS dementia complex) was the principal manifestation of cognitive, behavioral, and motor abnormalities in patients with the acquired immunodeficiency syndrome (AIDS). This has all but changed. Indeed, after introduction of highly active antiretroviral therapy (HAART) in 1996 and1997, the prevalence of severe manifestations of NeuroAIDS was significantly reduced, and the HIV patients show reduced disease morbidities and mortality. According to the Centers for Disease Control, the number of people 50 years and older infected with HIV has increased from $77 \%$ from 2001 to 2005. As HIV disease becomes a treatable medical disorder, morbidities in the HAART era abound including those linked to antiretroviral toxicities and viral infection per se as well as common disorders that all aging patients face, such as chronic obstructive pulmonary disease, diabetes, atherosclerosis-linked heart, vascular and kidney failure, bleeding ulcers, depression, cancers, and osteoporosis. Recent studies on aged HIV patient brain suggest a new type of neurological disorders distinct from HIV-1-associated

Electronic supplementary material The online version of this article (doi:10.1007/s11481-009-9155-5) contains supplementary material, which is available to authorized users.

T. Ikezu $(\bowtie)$

Department of Pharmacology and Experimental Neuroscience,

Center for Neurodegenerative Disorders,

University of Nebraska Medical Center,

985880 Nebraska Medical Center,

Omaha, NE 68198-5880, USA

e-mail: tikezu@unmc.edu dementia (HAD) and now termed HIV-associated neurocognitive disorders (HAND). HAND in aged population is linked, in part, to early signs of beta-amyloidosis or microtubule-associated tau aggregation commonly seen in Alzheimer's disease (AD) or tauopathy brain. However, whereas extracellular amyloid plaques (senile plaques) are the major amyloid pathology in AD, HAND brains show intraneuronal amyloid accumulation or perivascular diffuse amyloid deposition, indicating distinct mechanisms and brain disease development. Considering the increase in the aged HIV population, this issue of the Journal of Neuroimmune Pharmacology will specifically focus for the first time on age-associated neurodegeneration in HIV patients. Special emphasis was made on beta-amyloidosis contributed by an outstanding line of seven investigators representing the cutting edge of HAND research activities. Peer-reviewed original research and review articles provide clear direction for forthcoming HAND research directives.

The issue is lead by a comprehensive review of neurodegeneration and aging in the HAART era (Brew et al. 2009). This review discusses the history of NeuroAIDS, HAART, and persistent neurodegeneration in HAARTtreated patients. The implication of neurodegeneration in post-HAART patients is provocative and highlighted in its association with HIV, aging, and inflammation. Potential mechanisms common to specific diseases, such as AD and Parkinson's disease, and therapeutic inventions are proposed.

HAND pathogenesis, in the context of selective vulnerability of specific neuronal populations, epidemiology, and risk factors, is reviewed by Jayadev and Garden (2009). This article discusses a variety of host and viral factors that are associated with increased risk of developing HAND, and some of which are associated with the development of AD. Here, they propose a different view of how viral 
infection induces neurodegeneration and its common mechanism with $\mathrm{AD}$ pathogenesis.

Achim et al reports on intraneuronal accumulation of amyloid-beta $(A \beta)$ in HAART-treated aged HIV brains ranging in age from 38-60 (Achim et al. 2009). Autopsy findings now show more abundant $A \beta$ accumulation in HIV encephalitis (HIVE) brains than HIV-1 seropositive brains without encephalitis. This suggests that brain inflammation induced by viral infection can accelerate the intraneuronal $A \beta$ accumulation. This is among the first research articles demonstrating the links between HIV and neuroinflammation in the HAART era for HAND. The report shows that intraneuronal accumulation of amyloid can occur in the HIV-1-infected aged brain, partly similar to patients with AD.

This is followed by the study of Xu and Ikezu (2008), which proposes accelerated beta-amyloidosis in HIV brain in the HAART era. We demonstrate perivascular diffuse amyloid deposition and intraneuronal $A \beta$ accumulation in HIVE brains and inhibition of $\mathrm{A} \beta$ degradation in HIVinfected human macrophages. Potential molecular mechanisms of reduced $A \beta$ clearance and enhanced $A \beta$ production in the context of viral infection and inflammatory reaction are now proposed.

Lynn Pulliam (2009) reports a novel potential mechanism for the neuropathogenesis of HAND. Here, the viral protein Tat suppresses $A \beta$ degradation through its inhibition of neprilysin, an $A \beta$-degrading enzyme in neurons. This correlates with increased levels of $A \beta$ in the HIV-1-infected brain. In this report, the investigators demonstrate Tat-derived short peptide region responsible for the neprilysin inhibition. Moreover, the novel mechanism of MCP-1/CCL2 chemokine-mediated enhancement of $\mathrm{A} \beta$ production from neurons was developed in the context of enhanced brain beta-amyloidosis.

Tatro et al. provide a different aspect of complication in HAART era, a major depressive disorder (MDD), for its relationship with differential expression of FKBP51 and 52, immunophilins implicated in glucocorticoid receptor function and psychiatric disorders as a full research article (Tatro et al. 2009). This is a highly comprehensive neuropathological and genetic analysis of 59 cases. HIV patients with MDD show higher expression of FKBP52 (FKBP4) and FKBP51 (FKBP5) in frontal cortices. The group also reports an association of FKBP5 rs3800373 CC single nucleotide polymorphisms (SNP) with MDD and MDD/Psychosis groups, suggesting the role of SNP in 3' untranslated region of FKBP5 in MDD development.

Finally, Venneti et al. provide an excellent review on the role of microglia in neuroinflammation, neurodegeneration, immunotherapy, and the use of $\left[{ }^{11} \mathrm{C}\right]$ (R)-PK11195 for positron emission tomography (PET) imaging of microglia activation in human brains (Venneti et al. 2008). PK11195 is a ligand that binds to the translocator protein-18 expressed in microglia. As microglia play a key role as viral reservoirs, $A \beta$ clearance, and neuroinflammation, clinical in vivo imaging of microglia activation will be a useful tool. Alternatives to PK11195 for their application in $\mathrm{HIV}$ and $\mathrm{AD}$ brain imaging were discussed.

All together, these exciting review and research articles clearly highlight how neurodegenerative disorders, particularly beta-amyloidosis-related pathogenesis, are potentially prevalent in aged HAART-treated HIV patients. It is our hope that by gathering basic and clinical scientists with the common interest in HAND, the papers included will provide a platform for thought, discussion, and future basic, clinical, and translational research. More studies will come out from not only these laboratories but also many others in the HIV and neurodegenerative disorder research fields.

Acknowledgments We thank Dr. Howard E. Gendelman, Editor-inChief, and Robin Taylor, Managing Editor, for their invaluable support in counseling, organizing and editing this special issue. I thank each of the authors for their extraordinary contributions and my own laboratory members for their continuous support.

\section{References}

Achim CL, Adame A, Dumaop W, Everall IP, Masliah E (2009) Increased accumulation of intra-neuronal amyloid beta in HIVinfected patients J Neuroimmune Pharmacol. doi:10.1007/ s11481-009-9152-8.

Black R, Sager P (1994) Summary of workshop contributions. Third Workshop on NeuroAIDS. Adv Neuroimmunol 4:149156.

Brew BJ, Crowe SM, Landay A, Lucette AL, Guillemin G (2009) Neurodegeneration and ageing in the HAART era. J Neuroimmune Pharmacol (this issue). doi:10.1007/s11481-008-9143-1.

Jayadev S, Garden GA (2009) Host and viral factors influencing the pathogenesis of HIV associated neurocognitive disorders. J Neuroimmune Pharmacol. doi:10.1007/s11481-009-9154-6.

Pulliam L (2009) HIV regulation of amyloid beta production. J Neuroimmune Pharmacol. doi:10.1007/s11481-009-9151-9.

Tatro ET, Everall IP, Masliah E, Hult BJ, Lucero G, Chana G, Soontornniyomkij V, Achim CL (2009) Differential expression of immunophilins FKBP51 and FKBP52 in the frontal cortex of HIV-infected patients with major depressive disorder. J Neuroimmune Pharmacol. doi:10.1007/s11481-009-9146-6.

Venneti S, Wiley CA, Kofler J (2008) Imaging microglial activation during neuroinflammation and Alzheimer's disease. J Neuroimmune Pharmacol. doi:10.1007/s11481-009-9142-2.

Xu J, Ikezu T (2008) The comorbidity of HIV-associated neurocognitive disorders and Alzheimer's disease: a foreseeable medical challenge in post-HAART era. J Neuroimmune Pharmacol. doi:10.1007/ s11481-008-9136-0. 